\title{
Impact of Variable Thermal and Thermomechanical Environment on Graded FGM Square Plate
}

\author{
Manish Bhandari \\ Assistant Professor, Department of Mechanical Engineering, JNV University, Jodhpur, (Rajasthan), INDIA
}

\begin{abstract}
The functionally graded material (FGM) are combination of ceramic and metal and hence exhibit properties such that FGM becomes suitable for the conditions where high temperature environment is prevailing. The combination of the FGM is governed by the material variation which is done by following certain laws of material distribution such as Power law, Sigmoid law, Exponential law etc. The analysis of FGM under thermal and thermomechanical loadings have been important aspect for scientists in emerging areas of research. It is important to have response of FGM plates in the conditions of thermal and thermomechanical environment. In the current work, FEM is used to model FGM plate and non dimensional deflection, stress and strain are computed in the condition of variable thermal environment. The results are compared for PFGM, S-FGM and E-FGM.
\end{abstract}

Key words: FGM;Deflection; Stress;Strain; Thermomechanical.

\section{INTRODUCTION}

The functionally graded material (FGM) are combination of ceramic and metal and hence exhibit properties such that FGM becomes suitable for the conditions where high temperature environment is prevailing. The combination of the FGM is governed by the material variation which is done by following certain laws of material distribution such as Power law, Sigmoid law, Exponential law etc. The analysis of FGM under thermal and thermomechanical loadings have been important aspect for scientists in emerging areas of research. Suresh and Mortensen [1] computed deformations of FGM plate under thermo-mechanical environment and found geometrical and load situations responsible for in equilibrium, change in shape changes and bifurcation. Power law distribution is considered by Marcelo [3], Sigmoid distribution and Exponential distribution have been utilized by Bhavani [4]. Qian and Batra [6] worked on unsteady deformations under heating conditions for FG thick plate under thermal load and mechanical load on ceramic surface. Liviu and Daniel [8] solve problems of 2-D heat conduction for isotropic, orthotropic, single, composite and non-linear FGMs by deriving continuity conditions considering temperature and heat flux at the interfaces. Hui and Qing [9] created a mesh-less algorithm to conduct analysis for two-dimensional (2D) thermal and mechanical problems in FGMs. He showed that suitable parameter for grading will lead to lower concentration of stresses and lesser change in distribution of stress fields. Prakash [10] formulated Laplace transformations to derive differentials of unsteady heat transfer to differentials (ODE) along thickness direction to be solved using power series method. derived analytical solution in close form of an elliptic plate fixed at midplane in thermoelastic conditins. Nilanjan [4] reported that FGM plates exhibit high capability to sustain stresses under high temperature conditions. FGMs are reactive to flow of heat variation through structure, as compared to that of structures made of pure materials. Ashraf and Daoud [12] performed investigation of thermal deflection analysis for FGM plates subjected to uniform, linear and non-linear thermal loading along thickness and critical buckling temperature difference was found proportional to the plate aspect ratio. Mostaphaet. al [13] showed buckling analysis of FGM plate under thermal loading and found closed form solutions for the critical buckling temperatures of plates. Srinivas G et.al. [14] concluded that variation in stresses and deflection under thermo-mechanical loading is controlled by gradual variation in material properties.Dai et. al.[7] and Alshorbagy et. al.[15] derived equations dependent on the combination of the first order plate theory and the Von Karman strains. The Von Karman plate theory cosiders for moderately large deflections and small strains. It is assumed that transverse strain components are very less as compared to other strain components. Bhandari and Purohit [16] studied FGM plate when subjected to different types of mechanical loadings e.g. point load and uniform distributed loading. Qian and Batra [6] considered Aluminum- Silicon Carbide FGM, Reddy [2] and Bhandari and Purohit [17,18] used Aluminum-Zirconia FGM, Ashraf and Daoud [12] studied Aluminum-Alumina as FGM. Sharma [19] disclosed that material gradation affects the stability and failure behavior of FGM plate at a great level and concluded that FGM plate with elastic material properties exhibits stable equilibrium path. Xiaohui [20] analyzed the 
thermomechanical behaviors of FGM plates with various configurations and presented intensity of the effect of material gradient and thermal field on response of plates made of FGM..Senthil and Batra [5], Mostaphaet. al. [13] used uncoupled quasi-static thermo-elasticity theory. They found equilibrium and stability equations of a rectangular plate made of functionally graded material (FGM) under thermal loads based on the higher order shear deformation plate theory. Hamza et. al. [21] used latest engineering techniques for thermal analysis.

It is important to have response of FGM plates in the conditions of thermal and thermomechanical environment. In the current work, FEM is used to model FGM plate and non dimensional deflection, stress and strain are computed in the condition of variable thermal environment. The results are compared for P-FGM, S-FGM and E-FGM.

\section{METHODOLOGY}

A square $(1 \mathrm{mx} 1 \mathrm{~m})$ FGM plate is considered with a boundary condition of simply supported. Plate is made up Aluminum (Al) -Zirconia $\left(\mathrm{ZrO}_{2}\right)$. Thermal and thermo-mechanical analysis is performed. The thickness of the plate (h) is taken $0.02 \mathrm{~m}$.

\section{$2.1 \quad$ Thermal analysis}

Thermal analysis is performed by applying thermal environment to the FGM plate. At ceramic top surface temperature is varied form $50^{\circ} \mathrm{C}$ to $400^{\circ} \mathrm{C}$, metal surface is maintained at $0^{\circ} \mathrm{C}$ along with other edges.

\subsection{Thermomechanical analysis}

Analysis under thermomechanical conditions is conducted by providing thermal environment to the FGM plate alongwith a mechanical udl $\left(\mathrm{p}_{\mathrm{o}}\right)$ of $1 \mathrm{MPa}$. Temperature of $100{ }^{\circ} \mathrm{C}$ is applied at ceramic top surface while bottom (metal) surface and all edges are maintained at a temperature of $0{ }^{\circ} \mathrm{C}$.

Volume fraction exponent (n) is varied for the purpose of analysis in Power law-FGM, Sigmoid law-FGM and Exponential law-FGM e.g. for $\mathrm{n} ; 0$ (pure ceramic), n; $\infty$ (pure metal), n; 0.5, 2 for Power-FGM and Sigmoid- FGM and Exponential-FGM. Resultsare computed such as deflection $\left(\mathrm{u}_{\mathrm{z}}\right)$, tensile stress $\left(\sigma_{\mathrm{x}}\right)$, shear stress $\left(\sigma_{\mathrm{xy}}\right)$, strain $\left(\mathrm{e}_{\mathrm{x}}\right)$ and shear strain $\left(\mathrm{e}_{\mathrm{xy}}\right)$. Results are presented in non-dimensionalized outcomes i.e. non-dimensionalized deflection $\left(\overline{\mathrm{u}}_{\mathrm{z}}=\mathrm{u}_{\mathrm{z}} / \mathrm{h}\right)$, nondimensionalized tensile stress $\left(\overline{\sigma_{x}}=\sigma_{x} / p_{o}\right)$, non-dimensionalized shear stress $\left(\overline{\sigma_{x y}}=\sigma_{x y} / p_{o}\right)$, Strain $\left(e_{x}\right)$ and Shear strain $\left(e_{x y}\right)$. Finite element modelling is applied and simulation is done in ANSYS.

\section{RESULTS}

\subsection{Variable thermal environment}

Non dimensional parameters are computed when the FGM plate is subjected to variable thermal environment where temperature is varied form $50{ }^{\circ} \mathrm{C}$ of $400{ }^{\circ} \mathrm{C}$ for square simply supported plate. Non dimensional parameters are depicted in Tables 1,2,3,4 and 5. Graphical comparisons have presented in Figs. 1,2,3,4 and 5 .

\subsubsection{Non-Dimensional Deflection $\left(\overline{\mathbf{u}_{\mathrm{z}}}\right)$}

Table 1: Non-dimensional deflection $\left(\overline{\mathrm{u}_{\mathrm{z}}}\right)$ under variable thermal environment

\begin{tabular}{|l|l|l|l|l|l|l|l|}
\hline $\begin{array}{c}\mathbf{T} \\
\left({ }^{\circ} \mathbf{C}\right)\end{array}$ & $\begin{array}{c}\text { Ceramic } \\
(\mathbf{n 0})\end{array}$ & $\begin{array}{c}\text { Power } \\
(\mathrm{n} \\
0.5)\end{array}$ & $\begin{array}{c}\text { Sigmoid } \\
(\mathrm{n} 0.5)\end{array}$ & $\begin{array}{c}\text { Power } \\
(\mathrm{n} 2)\end{array}$ & $\begin{array}{c}\text { Sigmoid } \\
(\mathrm{n} 2)\end{array}$ & Exp. & $\begin{array}{c}\text { Metal } \\
(\mathbf{n} \infty)\end{array}$ \\
\hline 50 & 0.24 & 0.12 & 0.07 & 0.16 & 0.10 & 0.15 & 0.26 \\
\hline 100 & 0.49 & 0.23 & 0.14 & 0.33 & 0.21 & 0.3 & 0.52 \\
\hline 150 & 0.73 & 0.37 & 0.23 & 0.49 & 0.31 & 0.45 & 0.78 \\
\hline 200 & 0.98 & 0.49 & 0.30 & 0.65 & 0.41 & 0.6 & 1.03 \\
\hline 250 & 1.22 & 0.61 & 0.38 & 0.81 & 0.52 & 0.75 & 1.29 \\
\hline 300 & 1.47 & 0.73 & 0.45 & 0.98 & 0.62 & 0.9 & 1.55 \\
\hline 350 & 1.71 & 0.86 & 0.53 & 1.14 & 0.72 & 1.05 & 1.81 \\
\hline 400 & 1.95 & 0.98 & 0.61 & 1.31 & 0.83 & 1.2 & 2.07 \\
\hline
\end{tabular}

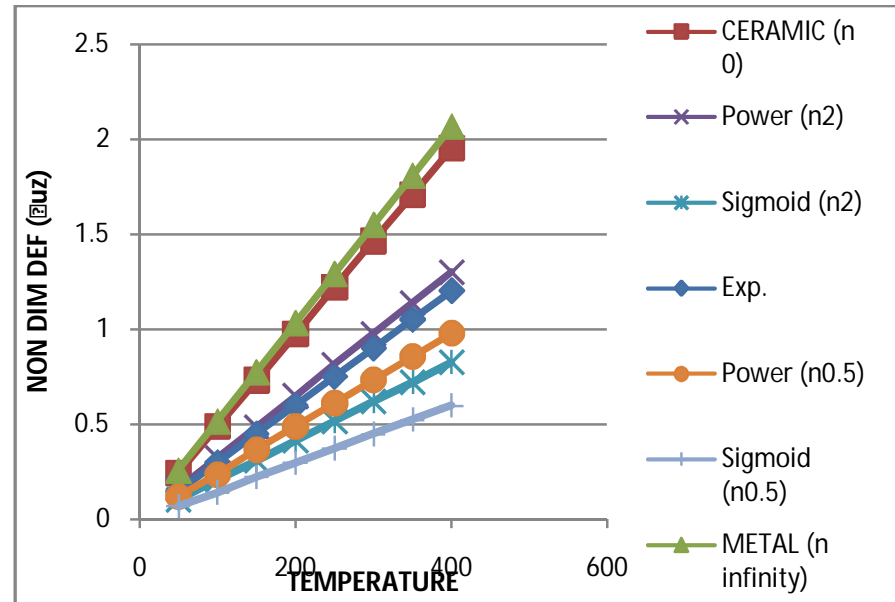

Figure 1: Non-dimensional deflection $\left(\overline{\mathrm{u}_{\mathrm{z}}}\right)$ under variable thermal environment

\subsubsection{Strain $\left(e_{x}\right)$}

Table 2: Strain $\left(\mathrm{e}_{\mathrm{x}} \mathrm{x} 1000\right)$ under variable thermal environment

\begin{tabular}{|l|l|l|l|l|l|l|l|}
\hline $\begin{array}{c}\mathbf{T} \\
\left({ }^{\circ} \mathbf{C}\right)\end{array}$ & $\begin{array}{c}\text { Ceramic } \\
(\mathbf{n ~ 0 )}\end{array}$ & $\begin{array}{c}\text { Power } \\
(\mathrm{n} \\
0.5)\end{array}$ & $\begin{array}{c}\text { Sigmoid } \\
(\mathrm{n} \text { 0.5) }\end{array}$ & $\begin{array}{c}\text { Power } \\
(\mathrm{n} 2)\end{array}$ & $\begin{array}{c}\text { Sigmoid } \\
(\mathrm{n} 2)\end{array}$ & Exp. & $\begin{array}{c}\text { Metal } \\
(\mathbf{n} \infty)\end{array}$ \\
\hline 50 & 0.65 & 0.18 & 0.34 & 0.43 & 0.40 & 0.29 & 0.67 \\
\hline 100 & 1.29 & 0.37 & 0.68 & 0.87 & 0.80 & 0.58 & 1.35 \\
\hline 150 & 1.94 & 0.55 & 1.02 & 1.3 & 1.20 & 0.87 & 2.02 \\
\hline 200 & 2.58 & 0.73 & 1.36 & 1.73 & 1.61 & 1.16 & 2.69 \\
\hline 250 & 3.23 & 0.92 & 1.70 & 2.17 & 2.01 & 1.44 & 3.36 \\
\hline 300 & 3.87 & 1.1 & 2.04 & 2.6 & 2.41 & 1.73 & 4.04 \\
\hline 350 & 4.52 & 1.28 & 2.38 & 3.03 & 2.81 & 2.02 & 4.71 \\
\hline 400 & 5.16 & 1.46 & 2.72 & 3.47 & 3.21 & 2.31 & 5.38 \\
\hline
\end{tabular}




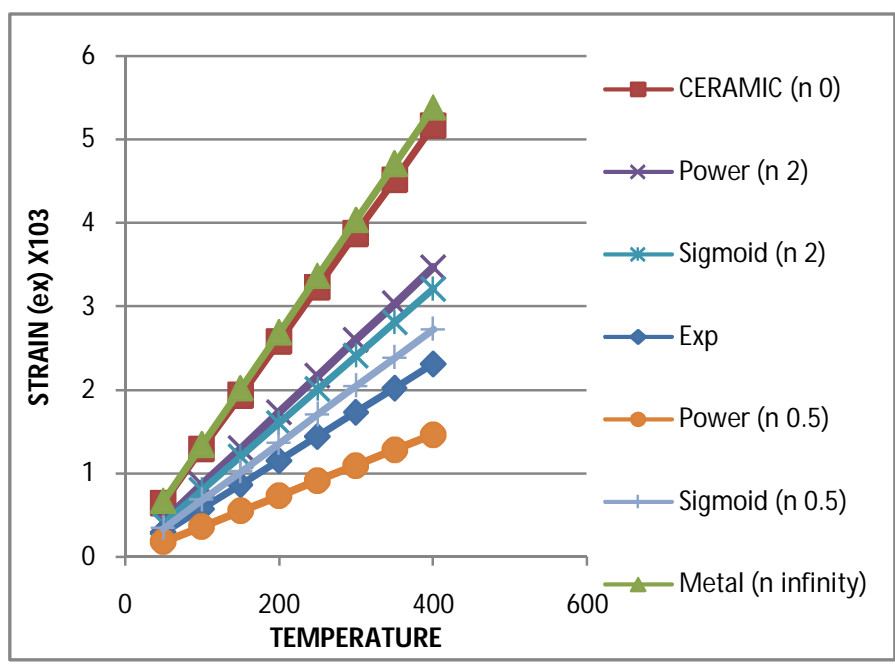

Figure 2: Strain $\left(\mathrm{e}_{\mathrm{x}}\right)$ under variable thermal environment

\subsubsection{Shear Strain $\left(\mathbf{e}_{\mathrm{xy}}\right)$}

Table 3: Shear strain $\left(\mathrm{e}_{\mathrm{xy}} \mathrm{x}\right.$ 1000)under variable thermal environment

\begin{tabular}{|l|l|l|l|l|l|l|l|}
\hline $\begin{array}{c}\mathbf{T} \\
\left({ }^{\circ} \mathbf{C}\right)\end{array}$ & $\begin{array}{c}\text { Cerami } \\
\mathbf{c} \\
(\mathbf{n} \mathbf{0})\end{array}$ & $\begin{array}{l}\text { Power } \\
(\mathrm{n} 0.5)\end{array}$ & $\begin{array}{c}\text { Sigmoi } \\
\mathrm{d}(\mathrm{n} \\
0.5)\end{array}$ & $\begin{array}{l}\text { Power } \\
(\mathrm{n} 2)\end{array}$ & $\begin{array}{l}\text { Sigmoi } \\
\mathrm{d}(\mathrm{n} 2)\end{array}$ & Exp. & $\begin{array}{l}\text { Metal } \\
(\mathbf{n} \infty)\end{array}$ \\
\hline 50 & 8.76 & 6.82 & 7.71 & 9.56 & 8.86 & 7.92 & 8.51 \\
\hline 10 & 20.92 & 13.6 & 15.43 & 18.0 & 17.72 & $\begin{array}{l}15.8 \\
4\end{array}$ & $\begin{array}{l}22.0 \\
0\end{array}$ \\
\hline 0 & & 5 & & 4 & & 4 & 0 \\
\hline 15 & 29.91 & 20.4 & 23.14 & 28.6 & 26.58 & $\begin{array}{l}23.7 \\
6\end{array}$ & $\begin{array}{l}32.7 \\
5\end{array}$ \\
\hline 0 & & 7 & & 7 & & 6 & 5 \\
\hline 20 & 39.88 & 27.3 & 30.86 & 38.2 & 35.44 & 31.6 & 43.6 \\
0 & & 0 & & 3 & & 9 & 7 \\
\hline 25 & 49.85 & 34.1 & 38.57 & 47.7 & 44.30 & 39.6 & 54.5 \\
0 & & 2 & & 8 & & 1 & 9 \\
\hline 30 & 59.81 & 40.9 & 46.28 & 57.3 & 53.16 & 47.5 & 65.5 \\
0 & & 5 & & 4 & & 3 & 1 \\
\hline 35 & 69.78 & 47.7 & 54.00 & 66.9 & 62.02 & $\begin{array}{l}55.4 \\
5\end{array}$ & $\begin{array}{l}76.4 \\
0\end{array}$ \\
\hline 40 & 79.75 & 54.6 & 61.71 & 76.4 & 70.88 & 63.3 & 87.3 \\
0 & & 0 & & 5 & & 7 & 4 \\
\hline
\end{tabular}

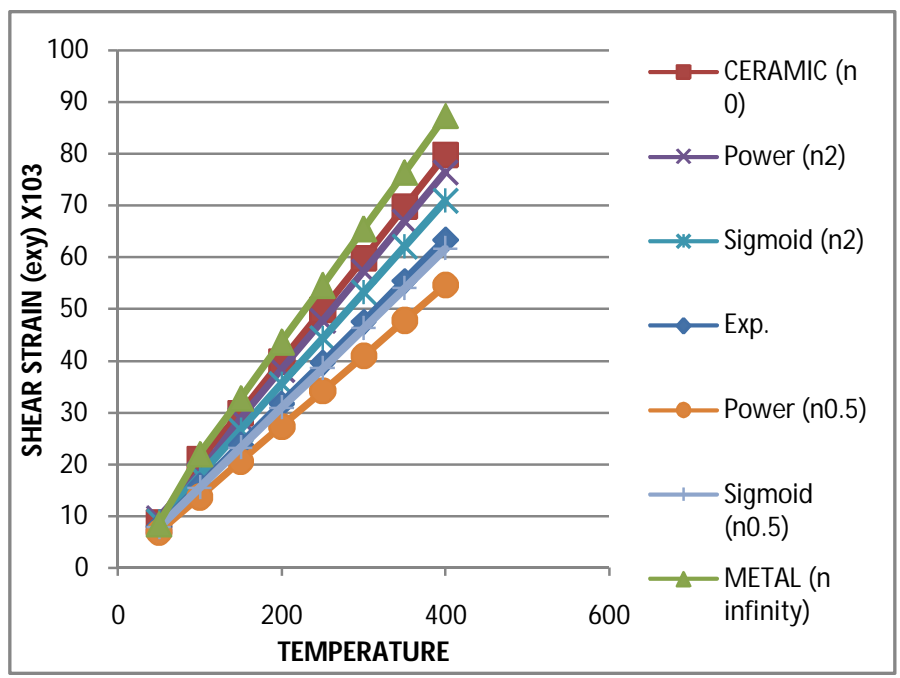

Figure 3: Shear strain $\left(e_{x y}\right)$ under variable thermal environment
The observations are as follows:

i. Deflection at $400^{\circ} \mathrm{C}$, for Power (n2) $\left(\overline{\mathrm{u}_{\mathrm{z}}}=1.31\right)$ is more that of Sigmoid (n2) $\left(\overline{\mathrm{u}_{\mathrm{z}}}=0.83\right)$. Deflection for Power $(\mathrm{n} 0.5)\left(\overline{\mathrm{u}_{\mathrm{z}}}=0.98\right)$ is more than Sigmoid $(\mathrm{n} 0.5)\left(\overline{\mathrm{u}_{\mathrm{z}}}=0.61\right)$ Deflection increases linearly with temperature.

ii. Tensile stress for Power (n2) $\left(\overline{\sigma_{\mathrm{x}}}=173\right)$ is more than Sigmoid (n2) $\left(\overline{\sigma_{\mathrm{x}}}=160.2\right)$. Tensile stress for Power (n0.5) $\left(\overline{\sigma_{\mathrm{x}}}=\right.$ $111.5)$ is lesser than Sigmoid $(\mathrm{n} 0.5)\left(\overline{\sigma_{\mathrm{x}}}=124.2\right)$. Tensile stress increases linearly with temperature.

iii. Shear stress for Power (n2) $\left(\overline{\sigma_{x y}}=2063\right)$ is more than Sigmoid (n2) $\left(\overline{\sigma_{x y}}=1910\right)$. Shear stress Power (n0.5) $\left(\overline{\sigma_{x y}}=\right.$ $1718)$ is less than Sigmoid (n0.5) $\left(\overline{\sigma_{x y}}=2078\right)$. Shear stress increases linearly with temperature.

iv. Strain $\left(e_{x}\right)$ for Power $(n 2)\left(e_{x}=3.47\right)$ is more than Sigmoid (n2) $\left(e_{x}=3.21\right)$. Also strain $\left(e_{x}\right)$ for Power $(n 0.5)\left(e_{x}=\right.$ 1.46) is lesser than Sigmoid $(n 0.5)\left(e_{x}=2.72\right)$. Strain increases linearly with temperature.

v. The shear strain $\left(e_{x y}\right)$ of Power (n2) $\left(e_{x y}=76.45\right)$ is more than Sigmoid $(n 2)\left(e_{x y}=70.88\right)$. Also shear strain for Power $(\mathrm{n} 0.5)\left(\mathrm{e}_{\mathrm{xy}}=54.6\right)$ is lesser than Sigmoid $(\mathrm{n} 0.5)\left(\mathrm{e}_{\mathrm{xy}}\right.$ $=61.71)$. Shear strain increases linearly with temperature.

vi. Deflection ofExp.FGM $\left(\overline{\mathrm{u}}_{\mathrm{z}}=1.2\right)$ isintermediate to Power (n2) $\left(\overline{\mathrm{u}_{\mathrm{z}}}=1.31\right)$ and Power $(\mathrm{n} 0.5)\left(\overline{\mathrm{u}_{\mathrm{z}}}=0.83\right)$.Same kind of observation is in other non-dimensionalized results.

\subsection{Variable thermal environment under mechanical load}

Non dimensional parameters are computed when the FGM plate is subjected to variable thermal environment where temperature is varied form $50{ }^{\circ} \mathrm{C}$ of $400{ }^{\circ} \mathrm{C}$ under constant udl of $1 \mathrm{MPa}$ for square simply supported plate. Non dimensional parameters are depicted in Tables 6,7,8,9 and 10. Graphical comparisons have presented in Figs. 6,7,8,9 and 10.

\subsubsection{Non-Dimensional Deflection $\left(\overline{\mathbf{u}_{\mathrm{z}}}\right)$}

Table 4: Non-dimensional deflection $\left(\overline{\mathrm{u}}_{\mathrm{z}}\right)$ under variable thermal environment and constant mechanical load

\begin{tabular}{|l|l|l|l|l|l|l|l|}
\hline $\begin{array}{c}\mathbf{T} \\
\left({ }^{\circ} \mathbf{C}\right)\end{array}$ & $\begin{array}{c}\text { Ceramic } \\
(\mathbf{n} \text { 0) }\end{array}$ & $\begin{array}{c}\text { Power } \\
(\mathrm{n} \\
0.5)\end{array}$ & $\begin{array}{c}\text { Sigmoid } \\
(\mathrm{n} \text { 0.5) }\end{array}$ & $\begin{array}{c}\text { Power } \\
(\mathrm{n} 2)\end{array}$ & $\begin{array}{c}\text { Sigmoid } \\
(\mathrm{n} 2)\end{array}$ & $\mathbf{E x p .}$ & $\begin{array}{c}\text { Metal } \\
(\mathbf{n} \infty)\end{array}$ \\
\hline 50 & 3.75 & 2.52 & 2.73 & 3.08 & 2.93 & 2.91 & 3.95 \\
\hline 100 & 3.9 & 2.64 & 2.84 & 3.24 & 3.13 & 3.06 & 4.03 \\
\hline 150 & 3.9 & 2.77 & 2.95 & 3.4 & 3.32 & 3.21 & 4.11 \\
\hline 200 & 3.94 & 2.89 & 3.06 & 3.57 & 3.51 & 3.36 & 4.15 \\
\hline 250 & 3.99 & 3.01 & 3.17 & 3.73 & 3.71 & 3.51 & 4.25 \\
\hline 300 & 4.04 & 3.13 & 3.29 & 3.89 & 3.9 & 3.66 & 4.45 \\
\hline 350 & 4.11 & 3.26 & 3.4 & 4.06 & 4.09 & 3.81 & 4.63 \\
\hline 400 & 4.2 & 3.38 & 3.51 & 4.22 & 4.19 & 3.96 & 4.83 \\
\hline
\end{tabular}


Manish Bhandari, International Journal of Emerging Trends in Engineering Research, 8(4), April 2020, 1005 - 1010

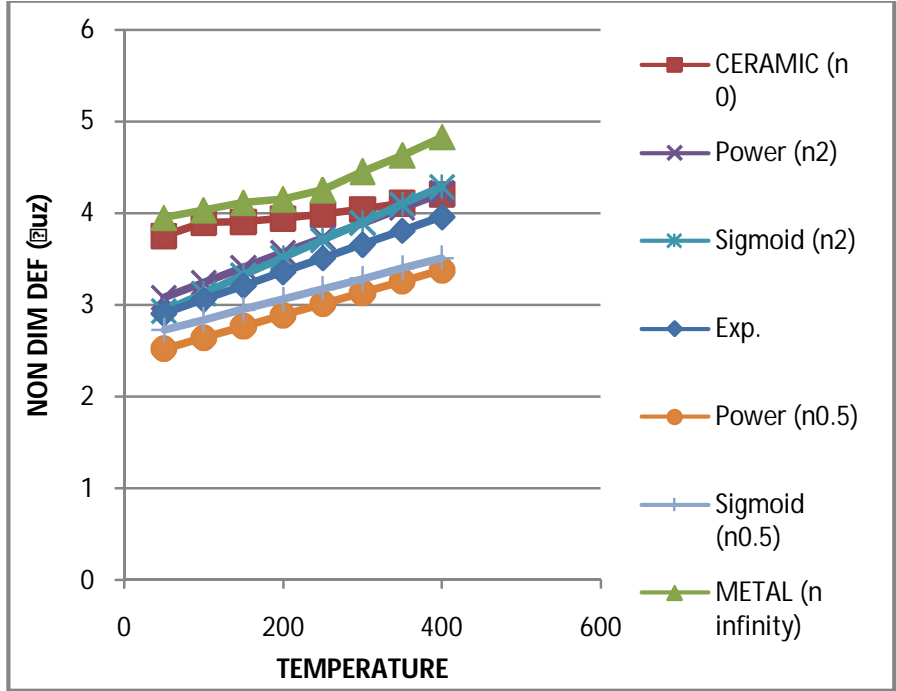

Figure 4: Non-dimensional deflection $\left(\overline{\mathrm{u}_{\mathrm{z}}}\right)$ under variable thermal environment and constant mechanical load

\subsubsection{Strain $\left(\mathbf{e}_{\mathrm{x}}\right)$}

Table 5: Strain $\left(\mathrm{e}_{\mathrm{x}} \mathrm{x}\right.$ 1000)under variable thermal environment and constant mechanical load

\begin{tabular}{|l|l|l|l|l|l|l|l|}
\hline $\begin{array}{c}\mathbf{T} \\
\left({ }^{\circ} \mathbf{C}\right)\end{array}$ & $\begin{array}{c}\text { Ceramic } \\
(\mathbf{n ~ 0 )}\end{array}$ & $\begin{array}{c}\text { Power } \\
(\mathrm{n} \\
0.5)\end{array}$ & $\begin{array}{c}\text { Sigmoid } \\
(\mathrm{n} \text { 0.5) }\end{array}$ & $\begin{array}{c}\text { Power } \\
(\mathrm{n} 2)\end{array}$ & $\begin{array}{c}\text { Sigmoid } \\
(\mathrm{n} 2)\end{array}$ & Exp. & $\begin{array}{c}\text { Metal } \\
(\mathbf{n} \infty)\end{array}$ \\
\hline 50 & 6.52 & 4.27 & 4.72 & 5.66 & 5.33 & 5.18 & 6.86 \\
\hline 100 & 6.30 & 3.83 & 4.31 & 5.33 & 4.98 & 4.77 & 6.46 \\
\hline 150 & 5.76 & 3.40 & 3.91 & 5.01 & 4.63 & 4.36 & 6.06 \\
\hline 200 & 5.37 & 2.98 & 3.52 & 4.73 & 4.32 & 3.96 & 5.66 \\
\hline 250 & 5.01 & 2.66 & 3.25 & 4.57 & 4.15 & 3.66 & 5.27 \\
\hline 300 & 4.72 & 2.43 & 3.07 & 4.55 & 4.09 & 3.45 & 4.97 \\
\hline 350 & 4.52 & 2.29 & 2.98 & 4.63 & 4.13 & 3.34 & 4.76 \\
\hline 400 & 4.40 & 2.21 & 2.98 & 4.80 & 4.27 & 3.31 & 4.63 \\
\hline
\end{tabular}

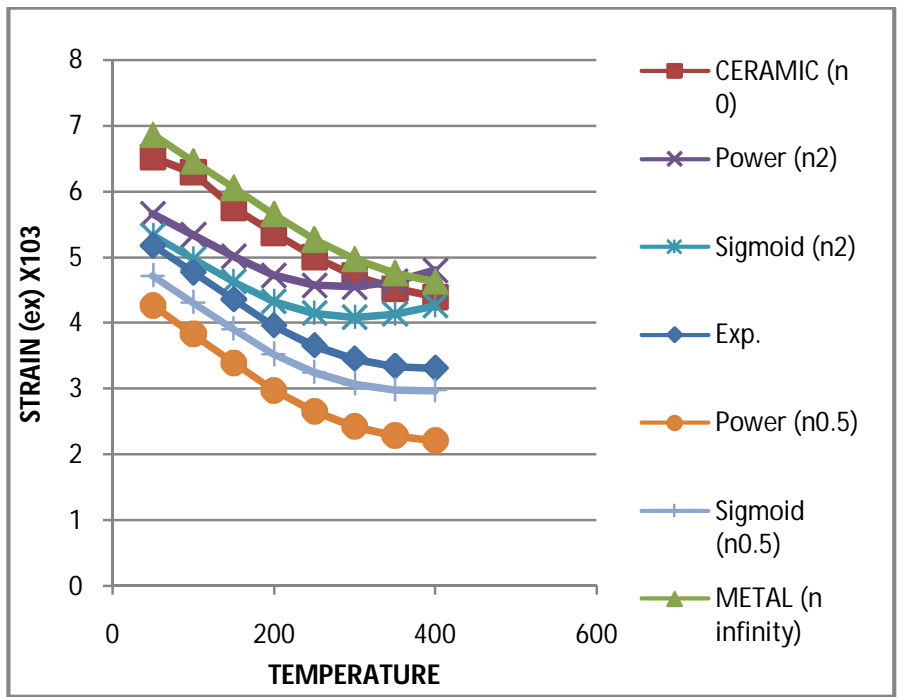

Figure 5: Strain $\left(e_{\mathrm{x}}\right)$ under variable thermal environment and constant mechanical load

\subsubsection{Shear Strain $\left(e_{x y}\right)$}

Table 6: Shear strain $\left(e_{x y} x 1000\right)$ under variable thermal environment and constant mechanical load

\begin{tabular}{|c|c|c|c|c|c|c|c|}
\hline $\begin{array}{c}\mathbf{T} \\
\left({ }^{\circ} \mathbf{C}\right)\end{array}$ & $\begin{array}{c}\text { Cerami } \\
\text { c } \\
(\mathbf{n} 0) \\
\end{array}$ & $\begin{array}{c}\text { Power } \\
\text { (n } \\
0.5) \\
\end{array}$ & $\begin{array}{c}\text { Sigmoi } \\
\text { d (n } \\
0.5) \\
\end{array}$ & $\begin{array}{c}\text { Power } \\
\text { (n 2) }\end{array}$ & $\begin{array}{l}\text { Sigmoi } \\
\mathrm{d}(\mathrm{n} 2)\end{array}$ & Exp. & $\begin{array}{l}\text { Metal } \\
(\mathbf{n} \infty)\end{array}$ \\
\hline 50 & 21.60 & $\begin{array}{l}15.8 \\
4\end{array}$ & 17.19 & $\begin{array}{l}20.1 \\
8\end{array}$ & 19.43 & $\begin{array}{l}18.4 \\
7\end{array}$ & $\begin{array}{l}22.7 \\
4\end{array}$ \\
\hline $\begin{array}{l}10 \\
0\end{array}$ & 27.95 & $\begin{array}{l}20.6 \\
1\end{array}$ & 22.39 & $\begin{array}{l}26.4 \\
5\end{array}$ & 25.46 & $\begin{array}{l}23.8 \\
4\end{array}$ & $\begin{array}{l}28.2 \\
2\end{array}$ \\
\hline $\begin{array}{l}15 \\
0\end{array}$ & 32.19 & $\begin{array}{l}25.3 \\
7\end{array}$ & 27.59 & $\begin{array}{l}32.7 \\
5\end{array}$ & 31.49 & $\begin{array}{l}29.2 \\
2\end{array}$ & $\begin{array}{l}33.8 \\
8\end{array}$ \\
\hline $\begin{array}{l}20 \\
0\end{array}$ & 37.57 & $\begin{array}{l}30.7 \\
6\end{array}$ & 34.53 & $\begin{array}{l}42.3 \\
1\end{array}$ & 39.56 & $\begin{array}{l}35.6 \\
4\end{array}$ & $\begin{array}{l}39.5 \\
5\end{array}$ \\
\hline $\begin{array}{l}25 \\
0\end{array}$ & 45.39 & $\begin{array}{l}37.5 \\
8\end{array}$ & 42.24 & $\begin{array}{l}51.8 \\
7\end{array}$ & 48.42 & $\begin{array}{l}43.5 \\
6\end{array}$ & $\begin{array}{l}47.7 \\
8\end{array}$ \\
\hline $\begin{array}{l}30 \\
0\end{array}$ & 53.47 & $\begin{array}{l}44.4 \\
1\end{array}$ & 49.95 & $\begin{array}{l}61.4 \\
2\end{array}$ & 57.28 & $\begin{array}{l}51.4 \\
8\end{array}$ & $\begin{array}{l}56.2 \\
8\end{array}$ \\
\hline $\begin{array}{l}35 \\
0\end{array}$ & 61.55 & $\begin{array}{l}51.2 \\
3\end{array}$ & 57.67 & $\begin{array}{l}70.9 \\
8\end{array}$ & 66.14 & $\begin{array}{l}59.4 \\
0\end{array}$ & $\begin{array}{l}64.7 \\
9\end{array}$ \\
\hline 40 & 69.64 & $\begin{array}{l}58.0 \\
6\end{array}$ & 65.38 & $\begin{array}{l}80.5 \\
4\end{array}$ & 75.00 & $\begin{array}{l}67.3 \\
2\end{array}$ & $\begin{array}{l}73.3 \\
0\end{array}$ \\
\hline
\end{tabular}

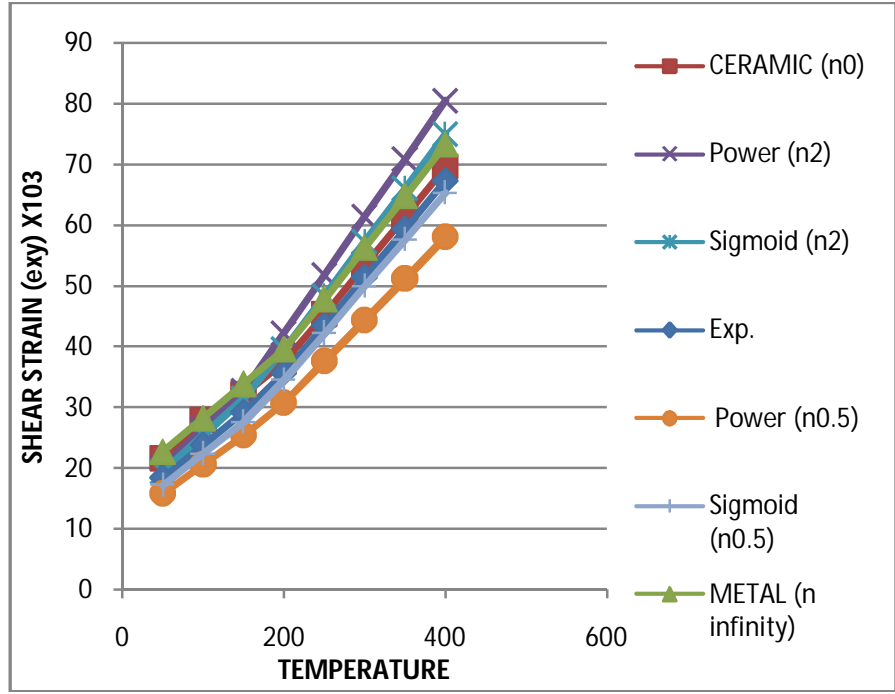

Figure 6: Shear strain $\left(\mathrm{e}_{\mathrm{xy}}\right)$ under variable thermal environment and constant mechanical load

The observations are as follows:

i. Deflection for Power $(\mathrm{n} 2)\left(\overline{\mathrm{u}_{\mathrm{z}}}=4.22\right)$ is more than Sigmoid (n2) $\left(\overline{\mathrm{u}_{\mathrm{z}}}=4.19\right)$. Deflection Power $(\mathrm{n} 0.5)\left(\overline{\mathrm{u}_{\mathrm{z}}}=3.38\right)$ is more than Sigmoid (n0.5) $\left(\overline{\mathrm{u}_{\mathrm{z}}}=3.51\right)$. The deflection increases with increase in temperature.

ii. Tensile stress for Power (n2) $\left(\overline{\sigma_{\mathrm{x}}}=341\right)$ is more than Sigmoid (n2) $\left(\overline{\sigma_{x}}=291\right)$. Tensile stress for Power $(\mathrm{n} 0.5)\left(\overline{\sigma_{x}}=139\right)$ is lesser than Sigmoid $(\mathrm{n} 0.5)\left(\overline{\sigma_{\mathrm{x}}}=208\right)$. Tensile stress shows decline with increase in temperature since the bending stress and thermal stress are of opposite nature in the case.

iii. Shear stress for Power (n2) $\left(\overline{\sigma_{x y}}=2173\right)$ is more than Sigmoid (n2) $\left(\overline{\sigma_{x y}}=2028\right)$. Also shear stress for Power (n0.5) 
$\left(\overline{\sigma_{\mathrm{xy}}}=1789\right)$ is less than Sigmoid $(\mathrm{n} 0.5)\left(\overline{\sigma_{\mathrm{xy}}}=1940\right)$. Shear stress is of incremental nature with temperature.

iv. Strain $\left(e_{\mathrm{x}}\right)$ for Power $(\mathrm{n} 2)\left(\mathrm{e}_{\mathrm{x}}=4.8\right)$ is more than Sigmoid (n2) $\left(e_{x}=4.27\right)$. Also strain $\left(e_{x}\right)$ for Power $(n 0.5)\left(e_{x}=2.21\right)$ is less than Sigmoid $(\mathrm{n} 0.5)\left(\mathrm{e}_{\mathrm{x}}=2.98\right)$. Strain shows decline with increase in temperature.

v. Shear strain $\left(\mathrm{e}_{\mathrm{xy}}\right)$ for Power $(\mathrm{n} 2)\left(\mathrm{e}_{\mathrm{xy}}=80.54\right)$ is more than Sigmoid $(\mathrm{n} 2)\left(\mathrm{e}_{\mathrm{xy}}=75\right)$. Also shear strain Power $(\mathrm{n} 0.5)\left(\mathrm{e}_{\mathrm{xy}}=\right.$ 58.36) is lesser than Sigmoid $(n 0.5)\left(e_{x y}=65.38\right)$. Shear strain is of incremental nature with temperature.

Non-dimensionalized deflection for Exp. FGM $\left(\overline{\mathrm{u}_{\mathrm{z}}}=4.83\right)$ is intermediate to Power (n2) $\left(\overline{\mathrm{u}_{\mathrm{z}}}=4.22\right)$ and Power $(\mathrm{n} 0.5)\left(\overline{\mathrm{u}_{\mathrm{z}}}=\right.$ 3.38).

\section{CONCLUSION}

A plate made of ceramic and metal which is called FGM has been studied under variable thermal and thermomechanical environment. Geometric parameters are computed by varying material distribution and temperature. The following conclusions are:

(a) Deflection for metal plate is more than FGM plates (i.e. $0<\mathrm{n}<\propto$ ). Ceramic and metal plates give least tensile stress as compared to various FGMs. The metal rich region and ceramic rich region gives comparable tensile stress. Shear stress diverge as volume fraction index increases.

(b) The minimum deflection, strain and stress is observed for Power FGM (n0.5) because stiffness of Power FGM (n0.5) plate is higher when compared to Exp. FGM plate and stiffness of Exp. FGM plate is higher when compared to that of Power FGM (n2).

The work may have extension in area of FG plates with more complex mechanisms such as variable mechanical and thermal loading combinations.

\section{REFERENCES}

[1]. Suresh S, Mortensen A, "Functionally Graded Metals and Metal-Ceramic", Composites Part 2 Thermo mechanical Behavior. Int Materials Reviews. Vol. 42, PP. 85-116, 1997. https://doi.org/10.1179/imr.1997.42.3.85

[2]. Reddy JN, "Analysis of Functionally Graded Plates", Int J Numer. Meth. Engg., Vol. 47, PP. 663-684, 2000.

[3]. Marcelo R. and Manuel D.L., "Homogeneity without Uniformity: Towards A Mathematical Theory of Functionally Graded Materials", .Int J of Solids and Structures, Vol. 37, PP. 7577-7591, 2000.

https://doi.org/10.1016/S0020-7683(99)00309-1

[4]. Bhavani V.S. and Jerome T.T., "Thermal Stresses in Functionally Graded Beams", AIAA J, Vol. 40, PP. 12281232, 2002.

https://doi.org/10.2514/3.15185

[5]. Senthil SV and Batra RC, "Three-Dimensional Analysis of Transient Thermal Stresses in Functionally Graded Plates",Int J of Solids and Structures, Vol. 40, PP. 7181-7196, 2003.

[6]. Qian LF and Batra RC, "Transient Thermoelastic Deformations of a Thick Functionally Graded Plate", J of Thermal Stresses, Vol. 27, PP. 705-740, 2004. https://doi.org/10.1080/01495730490440145

[7]. Dai KY, Liu GR, Han X, Lim KM., "Thermo mechanical Analysis of Functionally Graded Material Plates Using Element-Free Galerkin Method", Computers and Structures, Vol. 83, PP. 1487-1502, 2005.

https://doi.org/10.1016/j.compstruc.2004.09.020

[8]. Liviu M, Daniel L., "The Method of Fundamental Solutions for Nonlinear Functionally Graded Materials",Int J of Solids and Structures," Vol. 44, PP. 6878-6890, 2007.

https://doi.org/10.1016/j.ijsolstr.2007.03.014

[9]. Hui W, Qing-Hua Q, "Meshless Approach for Thermo-Mechanical Analysis of Functionally Graded Materials", Engg. Analysis with Boundary Elements, Vol. Vol. 32, PP. 704-712, 2008.

[10]. Prakash T, Singha MK, Ganapathi M., "Thermal Post buckling Analysis of FGM Skew Plates", Engg. Structures, Vol. 30, PP. 22-32, 2008.

https://doi.org/10.1016/j.engstruct.2007.02.012

[11]. Nilanjan C, Ravikiran K., "Comparative Analysis of Steady State Heat Transfer in A TBC and Functionally Graded Air Cooled Gas Turbine Blade",Indian Academy of Sciences, Vol. 35, PP. 1-17, 2010.

https://doi.org/10.1007/s12046-010-0006-0

[12]. Ashraf MZ, Daoud SM., "Thermal Buckling Analysis of Ceramic-Metal Functionally Graded Plates", Natural Science, Vol. 2, PP. 968-978, 2010.

https://doi.org/10.4236/ns.2010.29118

[13]. Mostapha R, Reza Al, Amirabbas K., "Thermal Buckling Of Thin Rectangular FGM Plate", World Applied Sciences J., Vol. 16, PP. 52-62, 2012.

[14]. Srinivas G, Shiva PU, Manikandan M, Praveen KA., "Simulation of Traditional Composites under Thermal Loads",Research J of Recent Sciences, Vol. 2, PP. 273-278, 2013.

[15]. Alshorbagy E, Alieldin SS, Shaat M, Mahmoud FF., “ Finite Element Analysis of The Deformation Of Functionally Graded Plates Under Thermomechanical Loads",Hindawi Publishing Corporation Mathematical Problems in Engg. 2013; PP. 1-14, 2013.

https://doi.org/10.1155/2013/569781

[16]. Bhandari M. and Purohit K., "Static Response of Functionally Graded Material Plate under Transverse Load for Varying Aspect Ratio", International Journal of Metals. [Online]Availablefrom

http://dx.doi.org/10.1155/2014/980563, 2014.

[17]. Bhandari M., Purohit K. and Sharma M., "Static Analysis of Functionally Gradient Material Plate with various Functions", Research Journal of Recent Sciences. Vol. 3(12), PP. 99-106, 2015.

[18]. Bhandari M. and Purohit K, "Response of Functionally Graded Material Plate under Thermomechanical Load Subjected to Various Boundary Conditions", International Journal of Metals,http://dx.doi.org/10.1155/2015/416824, 2015.

[19]. Sharma K. and Kumar D., "Elastoplastic Stability and Failure Analysis of FGM Plate with Temperature Dependent Material Properties under Thermomechanical Loading", Latin American Journal of Solids and Structures, Vol. 14, PP. 13611386, 2017. 
Manish Bhandari, International Journal of Emerging Trends in Engineering Research, 8(4), April 2020, 1005 - 1010

https://doi.org/10.1590/1679-78253747

[20]. Xiaohui R. and Zhen W, "A refined sinusoidal model for functionally graded plates subjected to thermomechanical loading", Journal of Composite Materials, vol. 2018, https://doi.org/10.1177/0021998318814158, 2018.

[21]. Hamza Marouani, Malika Tridane, El Mehdi Majdi, SoufianeZerraf, Mustafa Belhabra, Said Belaaouad, "Engineering Techniques applied for studies by Infrared vibration, crystallographic characterization and Thermal Behavior of two new cyclotriphosphates", IJETER, Vol. 8:1, PP. 239-246, 2020

https://doi.org/10.30534/ijeter/2020/30812020 